\title{
Defibrillation success in out-of-hospital cardiac arrest: Point in time of ventricular fibrillation recurrence after successful shock during early phase of cardiopulmonary resuscitation
}

\author{
Martin C. Sassen ${ }^{1}$, Dana M. Spies ${ }^{1}$, Jonathan Kiekenap ${ }^{1}$, Susanne Betz ${ }^{1}$, Clemens Kill ${ }^{2}$
}

${ }^{1}$ Center of Emergency Medicine, Philipps-University, Marburg, Germany

${ }^{2}$ Center of Emergency Medicine, University Hospital Essen, Essen, Germany

\section{Purpose of the study:}

In out-of-hospital cardiac arrest (OHCA) with ventricular fibrillation (VF) guidelines recommend defibrillation with biphasic shock [1], followed by chest compressions for two minutes before analyzing shock success. If then VF is detected again, it is unclear whether VF persisted or - after successful defibrillation - reoccurred within the twominutes-cycle of chest compressions [2].

We investigated the point in time of VF-recurrence.

\section{Materials and Methods:}

Between February 2014 and March 2018 we examined 185 consecutive shocks in a retrospective study of resuscitation attempts by ALS-ambulance in OHCA with initial VF (Marburg-Biedenkopf-County, Germany, 252,000 inhabitants). Three independent investigators analyzed ECG-recordings of the defibrillator Corpuls3 from the beginning of the CPR until two minutes after the third shock. Using filters from $2-10 \mathrm{~Hz}$ we reduced chest compression-artifacts. Successful shock was defined as VF-termination within $5 \mathrm{~s}$ after the shock (Figure 1.1) [3]. A relapse was defined as VF-recurrence in the interval between $5 \mathrm{~s}$ after a shock and the following shock (Figure 1.2). An unsuccessful shock was defined as persistent VF within $5 \mathrm{~s}$ after the shock (Figure 1.3).

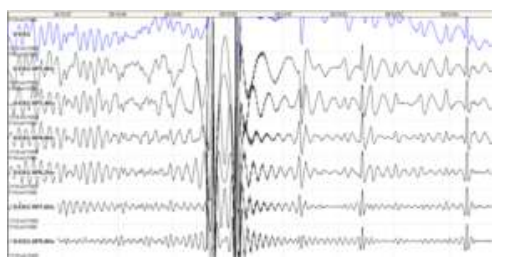

Figure 1.1: Return of organized rhythm

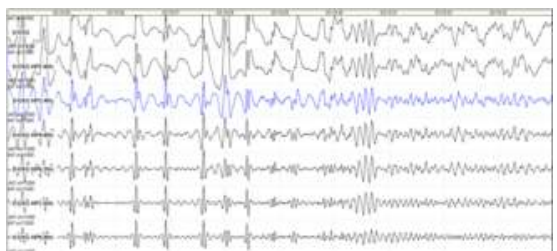

Figure 1.2: Relapse, recurrent VF

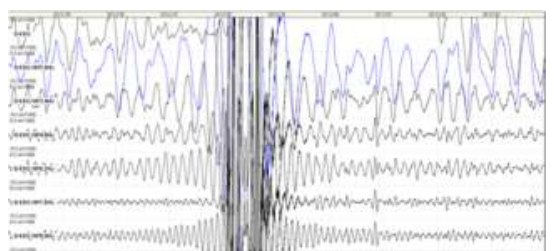

Figure 1.3: Persistent VF

\section{Results:}

We analyzed 185 shocks in 82 patients. $74.1 \%(n=137)$ of the shocks were successful, recurrence-rate of VF was 88.3\% ( $n=121$, Figure 2.1). The median of point in time of VF-recurrence was $33 \mathrm{~s}$ (16 s/106 s) s after shock. 47.1\% $(n=57)$ of VF-recurrence occurred $\leq 30 \mathrm{~s}$ after shock. $12.4 \%(n=15)$ of VF-recurrence occurred 31-60 s after shock. $13.7 \%(n=16)$ of VF-recurrence occurred $61-90 \mathrm{~s}$ after shock. $27.3 \%(n=33)$ of VF-recurrence occurred $>90 \mathrm{~s}$ after shock (Figure 2.2).

Figure 2.1: Defibrillation success

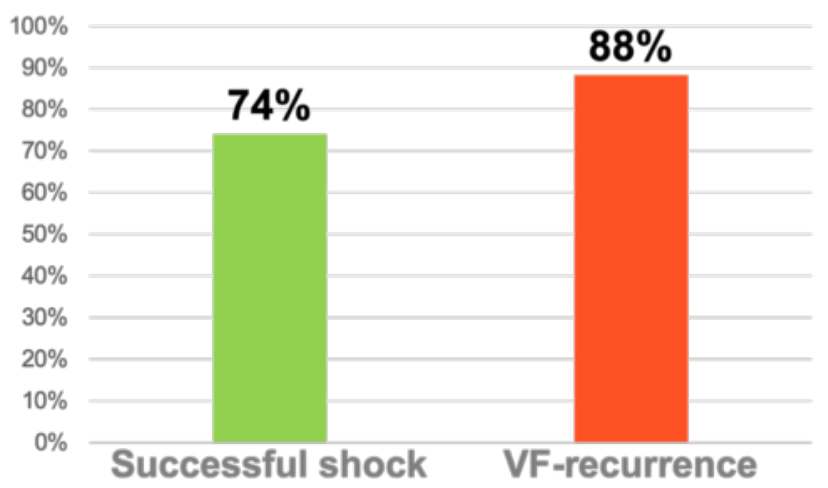

Figure 2.2: Point in time of VF-recurrence

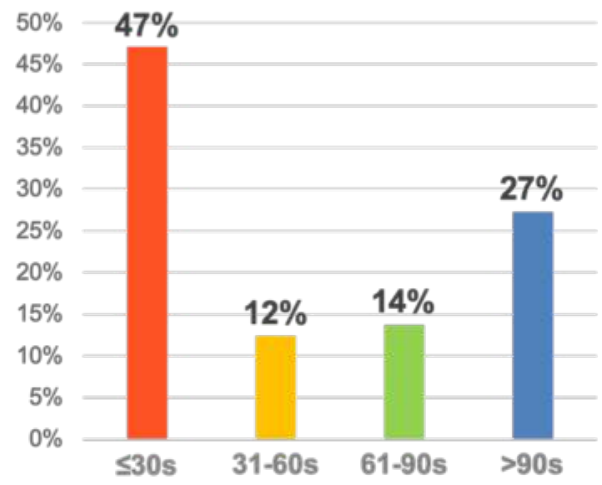

\section{Conclusions:}

Although VF was terminated by rectilinear-waveform-defibrillation in $74 \%$, VF recurred within two minutes of chest compressions in $88 \%$. Thus, VF reappears frequently and early. It is unclear to which extend chest compressions influence VF-relapse. Further studies need to reevaluate the best shock-compression-analysis-algorithm and the regime of antiarrhythmic therapy [4] in the early phase of CPR in OHCA with initial VF. 